\title{
Local Hamiltonian for Spherically Symmetric Gravity Coupled to a Scalar Field
}

\author{
Néstor Álvarez, ${ }^{1}$ Rodolfo Gambini, ${ }^{1}$ and Jorge Pullin ${ }^{2}$ \\ ${ }^{1}$ Instituto de Física, Facultad de Ciencias, Iguá 4225, esq. Mataojo, Montevideo, Uruguay \\ ${ }^{2}$ Department of Physics and Astronomy, Louisiana State University, Baton Rouge, Louisiana 70803-4001, USA
}

(Received 22 November 2011; published 30 January 2012)

We present a gauge fixing of gravity coupled to a scalar field in spherical symmetry such that the Hamiltonian is an integral over space of a local density. Such a formulation had proved elusive over the years. As in any gauge fixing, it works for a restricted set of initial data. We argue that the set could be large enough to attempt a quantization the could include the important case of an evaporating black hole.

Spherically symmetric gravity coupled to a scalar field has been an arena where many seminal ideas of black hole physics originated, through classical and semiclassical treatments. The full quantization of the model has resisted analysis, in part due to the complexity of the Hamiltonian structure of the system. If one could gain control of this model it would be a superb scenario to test key ideas about black hole evaporation. The first attempt to treat the problem quantum mechanically was carried out by Berger, Chitre, Nutku and Moncrief [1] and further developed by Unruh [2]. The resulting Hamiltonian was intractable enough that Unruh remarked "I present it here in the hope that someone else may be able to do something with it." More recently, Husain and Winkler, and Daghigh, Kunstatter and Gegenberg [3], using PainlevéGullstrand coordinates simplified somewhat Unruh's treatment. All these efforts, however, failed to produce a local Hamiltonian. We would like to show that using Ashtekar's new variables a gauge fixing can be found that yields a Hamiltonian that is the spatial integral of a Hamiltonian density. A similar gauge fixing can be carried out in traditional variables [4]. It also appears to apply in other $1+1$ models, like the Callan-Giddings-Strominger-Horowitz black holes [5]. We do not have a clear explanation as to why it seems to apply in such generality, it appears to be related to the possibility of defining a mass function $[6,7]$.

The subject of spherical symmetry with Ashtekar's new variables has been discussed in many instances. We will not carry out a full discussion here. We refer the readers to the literature. This is just a minimal introduction in order to make the Letter self-consistent. The topology of the spatial manifold will be chosen of the form $\Sigma=R^{+} \times S^{2}$. We will use a radial coordinate $x$ and study the theory in the range $[0, \infty]$. The case in which there is a horizon at $x=0$ can be treated with suitable boundary conditions.

Published by the American Physical Society under the terms of the Creative Commons Attribution 3.0 License. Further distribution of this work must maintain attribution to the author(s) and the published article's title, journal citation, and DOI.
The formalism for dealing with spherically symmetric gravity with Ashtekar's new variables was discussed by Bojowald and Swiderski[8] and also in our recent paper [9]. It is best to make several changes of variables to simplify things and improve asymptotic behaviors. We will not go through all these steps here. It suffices to notice that at the end of the process one is left with two pairs of canonical variables $E^{\varphi}$ and $K_{\varphi}$ (in our recent paper [9] called $\bar{A}_{\varphi}$ ), and $E^{x}$ and $K_{x}$, that are related to the traditional canonical variables in spherical symmetry

$$
d s^{2}=\Lambda^{2} d x^{2}+R^{2} d \Omega^{2}=\frac{\left(E^{\varphi}\right)^{2}}{E^{x}} d x^{2}+E^{x} d \Omega^{2}
$$

and $P_{\Lambda}=\sqrt{E^{x}} K_{\varphi} /(2 \gamma)$ where $\gamma$ is the Barbero-Immirzi parameter and $P_{\Lambda}$ is the momentum canonically conjugate to $\Lambda$, and we are considering the positive branch of $E^{x}$. One also has that the conjugate momentum to the variable $R$ is given by $P_{R}=\sqrt{E^{x}} \frac{K_{x}}{\gamma}+\frac{E^{\varphi} K_{\varphi}}{2 \sqrt{E^{x}} \gamma}$. The diffeomorphism and Hamiltonian constraint can be written as (To derive Eq. (3) one substitutes $\bar{A}_{\varphi}=2 \gamma K_{\varphi}, 2 \gamma K_{x}=A_{x}+\eta^{\prime}$ in equation (47) of Ref. [9] and adds the scalar field contribution, e.g., equation (2) of [10]. We have absorbed a factor of $4 \pi$ in Newton's constant.),

$$
\begin{gathered}
C_{x}=\frac{1}{G}\left[\left(E^{x}\right)^{\prime} K_{x}-E^{\varphi}\left(K_{\varphi}\right)^{\prime}\right]-P_{\phi} \phi^{\prime} \\
H=\frac{1}{G}\left[-\frac{1}{2} \frac{E^{\varphi}}{\sqrt{E^{x}}}-2 K_{x} K_{\varphi} \sqrt{E^{x}}-\frac{1}{2} \frac{K_{\varphi}^{2} E^{\varphi}}{\sqrt{E^{x}}}+\frac{1}{8} \frac{\left(E^{x}\right)^{\prime}}{\sqrt{E^{x}} E^{\varphi}}\right. \\
\left.-\frac{1}{2} \frac{\sqrt{E^{x}}\left(E^{x}\right)^{\prime}\left(E^{\varphi}\right)^{\prime}}{\left(E^{\varphi}\right)^{2}}+\frac{1}{2} \frac{\sqrt{E^{x}}\left(E^{x}\right)^{\prime \prime}}{E^{\varphi}}\right]+\frac{1}{2} \frac{P_{\phi}^{2}}{\sqrt{E^{x}} E^{\varphi}} \\
+\frac{1}{2} \frac{\left(E^{x}\right)^{3 / 2}\left(\phi^{\prime}\right)^{2}}{E^{\varphi}} .
\end{gathered}
$$

Recalling that the total Hamiltonian for the system is given by $H_{T}=\int d x\left(N^{x} C_{x}+N H\right)$, one can redefine the shift $N_{\text {new }}^{x}=N_{\text {old }}^{x}+2 N K_{\varphi} \sqrt{E^{x}} /\left(E^{x}\right)^{\prime}$, and the lapse $N_{\text {new }}=N_{\text {old }}\left(E^{x}\right)^{\prime} / E^{\varphi}$, and one gets a Hamiltonian constraint that reads, 


$$
\begin{aligned}
H= & \frac{1}{G}\left[-\frac{1}{2} \frac{\left(E^{x}\right)^{\prime}\left(1+K_{\varphi}^{2}\right)}{\sqrt{E^{x}}}+\frac{1}{8} \frac{\left(\left(E^{x}\right)^{\prime}\right)^{3}}{\left(E^{\phi}\right)^{2} \sqrt{E^{x}}}\right. \\
& -\frac{1}{2} \frac{\left(\left(E^{x}\right)^{\prime}\right)^{2} \sqrt{E^{x}}\left(E^{\varphi}\right)^{\prime}}{\left(E^{\varphi}\right)^{3}}+\frac{1}{2} \frac{\left(E^{x}\right)^{\prime} \sqrt{E^{x}}\left(E^{x}\right)^{\prime \prime}}{\left(E^{\varphi}\right)^{2}} \\
& \left.-2 K_{\varphi} \sqrt{E^{x}} K_{\varphi}^{\prime}\right]+\frac{1}{2} \frac{\left(\left(E^{x}\right)^{\prime}\right)^{2} P_{\phi}^{2}}{\left(E^{\varphi}\right)^{3} \sqrt{E^{x}}} \\
& +\frac{1}{2} \frac{\left.\left(\left(E^{x}\right)^{\prime}\right)^{2}\right)^{2}\left(E^{x}\right)^{3 / 2}\left(\phi^{\prime}\right)^{2}}{\left(E^{\varphi}\right)^{3}}-2 \frac{K_{\varphi} \sqrt{E^{x}} \phi^{\prime} P_{\phi}}{E^{\varphi}} .
\end{aligned}
$$

The quantity in the square brackets above is a total derivative,

$$
\begin{aligned}
H= & \frac{1}{G}\left[\frac{1}{4} \frac{\left(\left(E^{x}\right)^{\prime}\right)^{2} \sqrt{E^{x}}}{\left(E^{\varphi}\right)^{2}}-\left(K_{\varphi}^{2}+1\right) \sqrt{E^{x}}\right]^{\prime}-\frac{2 K_{\varphi} \sqrt{E^{x}} \phi^{\prime} P_{\phi}}{E^{\varphi}} \\
& +\frac{1}{2} \frac{\left(E^{x}\right)^{\prime} P_{\phi}^{2}}{\sqrt{E^{x}}\left(E^{\varphi}\right)^{2}}+\frac{1}{2} \frac{\left(E^{x}\right)^{\prime}\left(E^{x}\right)^{3 / 2}\left(\phi^{\prime}\right)^{2}}{\left(E^{\varphi}\right)^{2}} .
\end{aligned}
$$

This remarkable property is the key element in allowing to define a local Hamiltonian. Choosing a gauge in which the term involving the derivative does not depend on the gravitational variables, one is left with a Hamiltonian that only depends algebraically on the gravitational variables. As we mentioned, it appears that this is typical of all theories in $1+1$ dimensions that involve a mass function. It at least holds spherically symmetric Einstein gravity in the traditional and new variables, and for the CallanGiddings-Harvey-Strominger model.

We will completely gauge fix the theory. The first gauge condition is $\chi_{1}=0$ with

$$
\chi_{1}=E^{x}-x^{2} .
$$

In order to preserve the constraint in time the Lagrange multiplier $N^{x}$ gets fixed $N^{x}=0$. The diffeomorphism constraint can be solved, determining the variable $K_{x}$. The only constraint left is the Hamiltonian, which (omitting an overall factor $1 /\left(G\left(E^{\varphi}\right)^{2}\right)$ becomes,

$$
\begin{aligned}
H= & {\left[x\left(\frac{x^{2}}{\left(E^{\varphi}\right)^{2}}-K_{\varphi}^{2}-1\right)\right]^{\prime}\left(E^{\varphi}\right)^{2}-2 G x K_{\varphi} \phi^{\prime} P_{\phi} E^{\varphi} } \\
& +G P_{\phi}^{2}+G x^{4}\left(\phi^{\prime}\right)^{2} .
\end{aligned}
$$

Our strategy will be to perform a canonical transformation from the variables $\phi, P_{\phi}, K_{\varphi}, E^{\varphi}$ to a new set of variables $X, P_{X}, f, P_{f}$ such that $X$ is essentially what appears in the square brackets differentiated. We will later fix the gauge by setting $X$ equal to a given function of $t, x$. As a consequence $P_{X}$, the canonical momentum of $X$, will not appear differentiated in the constraint. This means that preserving the gauge fixing condition will lead to an algebraic equation that determines the lapse, and therefore to a local true Hamiltonian.

To construct the canonical transformation, let us start by identifying the variable $X$,

$$
-x\left(\frac{x^{2}}{\left(E^{\varphi}\right)^{2}}-K_{\varphi}^{2}-1\right)=X x^{2} \phi^{2}+2 G M(t) .
$$

Recalling that the scalar field has dimensions of inverse length in $3+1$ dimensions, the factor $x^{2}$ on the right is chosen so $X$ has dimensions of length (or time, since we chose $c=\hbar=1$ ), since it will later play the role of time. The factor $\phi^{2}$ is chosen so weak fields behave well in the gauge fixing (for instance if $\phi=0$ one has $K_{\varphi}=0$ and $E^{\varphi}=x / \sqrt{1-2 G M / x}$ in the usual Schwarzschild gauge). We added a function of time $M(t)$. Later, if one studies the fields asymptotically one finds that $M$ is a constant that corresponds to the Arnowitt-Deser-Misner mass. At the moment it is just a choice in the definition of $X$.

To complete the canonical transformation we then seek a generating function, we choose it to be of type I $F_{1}\left(\phi, K_{\varphi}, X, f\right)$, so one has that (recalling that $\left.\left\{K_{\varphi}(x), E^{\varphi}(y)\right\}=G \delta(x-y)\right)$,

$$
\begin{gathered}
G \frac{\partial F_{1}}{\partial K_{\varphi}}=E^{\varphi}, \\
\frac{\partial F_{1}}{\partial \phi}=P_{\phi}, \\
\frac{\partial F_{1}}{\partial f}=-P_{f}, \\
\frac{\partial F_{1}}{\partial X}=-P_{X} .
\end{gathered}
$$

We start from the first equation and note that we can use (8) to write $E^{\varphi}$ in terms of the quantities that the generating function depends on,

$$
E^{\varphi}=-\frac{x}{Y},
$$

where we chose the minus sign of the square root so the Hamiltonian is positive definite and for brevity we write,

$$
Y=\sqrt{K_{\varphi}^{2}+1-\frac{2 G M}{x}-x X \phi^{2}} .
$$

So we can now proceed to integrate (9) and choosing the integration constant to give the simplest form to the generating function yields, $F_{1}=-\frac{x}{G} \log \left(K_{\varphi}+Y\right)+\phi f$.

With the generating function and (10)-(12) we find the explicit form of the new variables in terms of the old ones,

$$
\begin{gathered}
P_{\phi}-\frac{x^{2} X \phi}{G Y\left(K_{\varphi}+Y\right)}=f, \\
P_{f}=-\phi, \\
P_{X}=-\frac{x^{2} \phi^{2}}{2 G Y\left(K_{\varphi}+Y\right)} .
\end{gathered}
$$


The last equation will become the Hamiltonian constraint when we rewrite the right-hand side entirely in terms of the new variables. Rewriting $\phi$ is immediate. To obtain $K_{\varphi}$ we solve (7) rewritten in terms of the new variables, i.e.,

$$
H=-\frac{\left(x^{2} \phi^{2} X\right)^{\prime}}{Y^{2}}+\frac{2 G x^{2} K_{\varphi} \phi^{\prime} P_{\phi}}{Y}+G P_{\phi}^{2}+G x^{4}\left(\phi^{\prime}\right)^{2},
$$

and $P_{\phi}$ and $\phi$ given by (15) and (16) respectively, so we have

$$
K_{\varphi}=\frac{x U^{2}-x+2 M G+X P_{f}^{2} x^{2}}{2 x U}
$$

with

$$
\begin{aligned}
U= & \frac{1}{\left(f+x^{2} P_{f}^{\prime}\right) \sqrt{x G}}\left(\left(P_{f}^{\prime}\right)^{2} x^{4} G Z+2 x^{5} P_{f}^{\prime} X P_{f}\right. \\
& \left.+2\left(X P_{f}^{2} x^{2}\right)^{\prime} x^{3}+2 X x^{3} P_{f} f+f^{2} G Z+2 x V^{1 / 2}\right)^{1 / 2},
\end{aligned}
$$

and

$$
\begin{gathered}
V=x\left(\left(P_{f}^{\prime}\right)^{2} x G Z+\left(X P_{f}^{2} x^{2}\right)^{\prime}\right)\left(f^{2} G Z+2 x^{3} X P_{f}\left(x^{2} P_{f}^{\prime}+f\right)\right. \\
\left.+\left(X P_{f}^{2} x^{2}\right)^{\prime} x^{3}\right) \\
Z=x^{2} X P_{f}^{2}+2 G M-x .
\end{gathered}
$$

We now consider (17) written entirely in terms of the new variables,

$$
P_{X}+\frac{x^{3} P_{f}^{2}}{2 G Y\left(f, P_{f}\right)\left[K_{\varphi}\left(f, P_{f}\right)+Y\left(f, P_{f}\right)\right]}=0 .
$$

This expression is the Hamiltonian constraint that is now easy to deparameterize. The total Hamiltonian is given by, $H_{\text {Total }}=\int d x N\left(P_{X}+\mathcal{H}_{\text {True }}\right)$, where we recognize the true Hamiltonian density,

$$
\mathcal{H}_{\text {True }}=\frac{x^{3} P_{f}^{2}}{2 G Y\left(f, P_{f}\right)\left[K_{\varphi}\left(f, P_{f}\right)+Y\left(f, P_{f}\right)\right]} .
$$

To prove that indeed this expression is the true Hamiltonian density, we proceed to completely fix the gauge. We choose $\chi_{2}=-X+g(x)+t=0$. The preservation in time of this condition, $\frac{\partial \chi_{2}}{\partial t}+\left\{\chi_{2}, H_{\text {Total }}\right\}=0$ implies that the lapse $N=1$. The system is now totally described in terms of the matter field variables $f, P_{f}$, since $X$ is fixed by the gauge fixing and $P_{X}$ is given by minus the true Hamiltonian. If we now consider the time evolution of the remaining variables,

$$
\begin{gathered}
\dot{f}=\left\{f, H_{\text {Total }}\right\}=\left\{f, H_{\text {True }}\right\}, \\
\dot{P}_{f}=\left\{P_{f}, H_{\text {Total }}\right\}=\left\{P_{f}, H_{\text {True }}\right\},
\end{gathered}
$$

showing that the true Hamiltonian indeed generates the evolution.

The expression for $K_{\varphi}$ (19) contains a series of square roots. This reflects the fact that the construction will not work for generic initial data, as one expects in gauge fixed treatments. In order to analyze under which conditions the construction works, we study the situation of weak fields, so we will assume $f=O(\epsilon)$ and $P_{f}=O(\epsilon)$ with $\epsilon \ll 1$ and we will keep only leading terms in $\epsilon$ in all equations. We will also assume that $M \sqrt{G} \gg 1$ (we are using units where $\hbar=c=1$ ). In order to simplify expressions we will also assume $g(x)=c x$ with $c$ a positive constant. The expression for $U$ becomes,

$$
\begin{aligned}
U= & \frac{1}{f+x^{2} P_{f}} \sqrt{\frac{2 r}{G}}\left(\left(x^{3} c P_{f}^{2}\right)^{\prime}+c x f P_{f}+x^{3} P_{f}^{\prime} c P_{f}\right. \\
& \left.+\left\{\left(x^{3} c P_{f}^{2}\right)^{\prime}\left[2 c x f P_{f}+\left(x^{3} c P_{f}^{2}\right)^{\prime}+2 x^{3} P_{f}^{\prime} c P_{f}\right]\right\}^{1 / 2}\right)^{1 / 2} .
\end{aligned}
$$

Sufficient conditions for the existence of the square roots are,

$$
\begin{gathered}
\left(x^{3} c P_{f}^{2}\right)^{\prime}=w(x), \\
\left(2 c x f P_{f}+2 x^{3} P_{f}^{\prime} c P_{f}\right)=v(x),
\end{gathered}
$$

with $w(x)$ and $v(x)$ positive functions. Solving the differential equations we get,

$$
\begin{gathered}
P_{f}=\frac{\sqrt{x \int^{x} w\left(x^{\prime}\right) d x^{\prime}}}{x^{2}} \\
f=\frac{3 x \int^{x} w\left(x^{\prime}\right) d x^{\prime}-x^{2} w(x)}{2 x^{3} P_{f}(x)}+\frac{v(x)}{x} .
\end{gathered}
$$

So we see that indeed one can specify initial data in the gauge we chose.

We have therefore presented for the first time a local Hamiltonian for a scalar field coupled to gravity in spherical symmetry, a problem that was unclear had a solution. The technique appears applicable in other $1+1$ dimensional situations where there exists a mass function. The result has a counterpart in path integral treatments, where authors were able to integrate out the gravitational variables [11]. This includes the Callan-Giddings-HorowitzStrominger model, which has received renewed attention recently [12] and is one of the best understood models of black hole evaporation. In further work we will discuss the boundary treatment in these coordinates and will show the evolution of collapsing scalar field pulses numerically. The resulting unconstrained system can be useful for quantization in situations involving gravitational collapse and black hole evaporation.

We wish to thank Daniel Grumiller, Viqar Husain, Gabor Kunstatter and especially Bill Unruh for comments, and a 
referee for corrections. This work was supported in part by Grant NSF-PHY-0968871, funds of the Hearne Institute for Theoretical Physics, CCT-LSU, Pedeciba and ANII PDT63/076. This publication was made possible through the support of a grant from the John Templeton Foundation. The opinions expressed in this publication are those of the author(s) and do not necessarily reflect the views of the John Templeton Foundation.

[1] B. Berger, D. Chitre, Y. Nutku, and V. Moncrief, Phys. Rev. D 5, 2467 (1972).

[2] W. Unruh, Phys. Rev. D 14, 870 (1976).

[3] V. Husain and O. Winkler, Phys. Rev. D 71, 104001 (2005); R. G. Daghigh, G. Kunstatter, and J. Gegenberg, Classical Quantum Gravity 24, 2099 (2007).

[4] W. Unruh (private communication).
[5] C. G. Callan, Jr., S. B. Giddings, J. A. Harvey, and A. Strominger, Phys. Rev. D 45, R1005 (1992).

[6] K. V. Kuchar, Phys. Rev. D 50, 3961 (1994).

[7] J. Gegenberg, G. Kunstatter, and D. Louis-Martinez, Phys. Rev. D 51, 1781 (1995).

[8] M. Bojowald and R. Swiderski, Classical Quantum Gravity 23, 2129 (2006).

[9] M. Campiglia, R. Gambini, and J. Pullin, Classical Quantum Gravity 24, 3649 (2007).

[10] V. Husain, in Proceedings of the Conference in Honor of C. N. Yang's 85th Birthday, edited by M.-L. Ge, C. H. Oh, and K. K. Phua (World Scientific, Singapore, 2008).

[11] D. Grumiller, W. Kummer, and D. V. Vassilevich, Phys. Rep. 369, 327 (2002); L. Bergamin, D. Grumiller, and W. Kummer, J. High Energy Phys. 05 (2004) 060; D. Grumiller and R. Meyer, Classical Quantum Gravity 23, 6435 (2006).

[12] A. Ashtekar, F. Pretorius, F. M. Ramazanoglu, Phys. Rev. Lett. 106, 161303 (2011); Phys. Rev. D 83, 044040 (2011). 